A N N A L E S Annales de Bretagne et des Pays de l'Ouest

\title{
Jacques Depauw, Spiritualité et pauvreté à Paris au XVIIe siècle
}

\section{Georges Provost}

\section{(2) OpenEdition \\ 1 Journals}

Édition électronique

URL : http://journals.openedition.org/abpo/1649

DOI : $10.4000 /$ abpo. 1649

ISBN : 978-2-7535-1485-0

ISSN : 2108-6443

Éditeur

Presses universitaires de Rennes

Édition imprimée

Date de publication : 20 mars 2002

Pagination : $173-176$

ISBN : 978-2-86847-708-8

ISSN : 0399-0826

\section{Référence électronique}

Georges Provost, "Jacques Depauw, Spiritualité et pauvreté à Paris au XVIIe siècle », Annales de Bretagne et des Pays de l'Ouest [En ligne], 109-1 | 2002, mis en ligne le 20 mars 2004, consulté le 23 septembre 2020. URL : http://journals.openedition.org/abpo/1649 ; DOI : https://doi.org/10.4000/ abpo.1649 
sentées : on y relève l'intéressante recherche de C. Grelet sur les conditions d'existence du bas-clergé poitevin au XVIII ${ }^{\mathrm{e}}$ siècle.

Ces actes sont aussi l'occasion de dégager des perspectives d'exploitation des sources d'histoire religieuse dans les années à venir. "Les possibilités sont encore grandes mais ce sera au prix de plus gros efforts ", avertit J. Marcadé. Nul doute que le constat soit valable en bien d'autres régions : l'historien du religieux devra tirer profit de sources judiciaires, notariales, etc., où le religieux ne sera pas directement accessible. L'inégalité de richesse entre les fonds ecclésiastiques d'un département à l'autre s'en trouvera peut-être partiellement réduite. Un regard au-delà des frontières permet, à ce propos, de mesurer tout le bénéfice de l'" exception française " de centralisation départementale des fonds ecclésiastiques : dans le diocèse d'Algarve au Portugal (B. Léal), la dispersion dans les presbytères est la règle, ce qui ne facilite pas, on s'en doute, l'appréhension, physique autant qu'intellectuelle, de la recherche. On n'oubliera pas, cependant, que la dispersion existe aussi en France, avec tous les risques qu'elle comporte : V. Adoumié le rappelle pour les archives post-concordataires, notamment celles des œuvres et des mouvements d'action catholique, et c'est avec inquiétude qu'il se demande " s'il sera possible demain de faire l'histoire religieuse d'aujourd'hui " : pour être chronologiquement marginale par rapport au reste du recueil, la question n'en vaut pas moins d'être posée à tous ceux qui en partagent, à un titre ou un autre, la responsabilité.

Georges Provost

DePAuw, Jacques, Spiritualité et pauvreté à Paris au XVII siècle, Paris, La Boutique de l'histoire, 1999, 363 p. (145 F)

Dans la riche historiographie née à partir des années soixante autour du problème de la pauvreté, la thèse désormais éditée de Jacques Depauw a l'originalité d'associer deux aspects qui ordinairement se rencontrent peu : l'un relevant de l'histoire religieuse - la vision spirituelle de la pauvreté - l'autre de l'histoire sociale - le traitement de la pauvreté sous forme d'assistance et/ou de renfermement - la rencontre s'opérant dans le cadre si riche du Paris du XVII siècle. L'originalité est d'autant plus nette que dans le couple, la spiritualité est première. Au long des cinq parties chronologiques qui jalonnent ce livre, c'est toujours elle qui ouvre la marche, véritable terreau où s'enracinent les diverses " politiques " d'assistance; à l'issue du parcours, c'est elle aussi qui ferme le ban. Un tel parti a imposé à l'auteur l'analyse de la surabondante littérature de piété publiée à Paris au fil du siècle : depuis les grands traités de spiritualité jusqu'aux petits recueils " populaires " de Noël en passant par les livres liturgiques. Sources arides, où la pauvreté apparaît souvent mais toujours de façon ambivalente : il y est question du pauvre " matériel " mais au moins aussi souvent du " pauvre en esprit " c'est-à-dire de l'attitude de tout pécheur humble et obéissant à l'Esprit, quelle que soit sa condition sociale. D'où la nécessité d'une rigoureuse analyse des textes, appuyée sur de généreuses citations. Mais cela ne dispense pas l'auteur de proposer également force tableaux, cartes et graphiques, issus de sources sérielles aujourd'hui bien connues : testaments, registres d'entrée et de comptabilité des hôpitaux, etc. C'est dire qu'il s'agit là d'un travail de très grande ampleur. 
L'auteur brosse d'abord la situation au début du XVII ${ }^{\mathrm{e}}$ siècle ( ${ }^{\text {re }}$ partie), caractérisée par un certain retard de Paris par rapport aux institutions charitables de Venise ou de Rome, où la Réforme catholique a déjà multiplié orphelinats et hôpitaux : Paris en reste à une poussière de petits établissements à l'ancienne, coiffée par un Bureau des pauvres issu de la réflexion humaniste des années 1530. "L'honnête homme un peu sceptique du lendemain des guerres de religion " est d'ailleurs peu sensible au problème de la pauvreté : avec Pierre Charron, il entend secourir l'affligé, mais "stoïquement ", sans céder à une excessive compassion envers le pauvre, "sotte et féminine pitié ".

C'est pourtant dès ce moment que prend naissance un " cycle de spiritualité active " ( $2^{\mathrm{e}}$ partie), à l'école des grands auteurs spirituels qui se taillent les grands succès d'édition dans le Paris des années 1610-1630. François de Sales, notamment, attire l'attention sur la pauvreté du laïc, distincte de la pauvreté volontaire du religieux : une pauvreté laïque qui est recommandable à la fois par sa propre valeur spirituelle et parce qu'elle offre à la quête de salut du dévot l'occasion d'une œuvre bénéfique entre toutes. D'où le vigoureux appel de l'Introduction à la vie dévote: "Allez les servir dans leur lit de vos propres mains, soyez leur cuisinière, leur lingère et leur blanchisseuse. " De pieux laïcs vont donc à la rencontre des pauvres : J. Depauw met en exergue la figure " héroïque " du " pauvre prêtre " Claude Bernard, pionnier méconnu antérieur à Vincent de Paul. La motivation des uns et des autres est avant tout d'ordre spirituel mais elle n'en débouche pas moins sur un certain nombre de fondations nouvelles, issues d'initiatives individuelles appuyées sur de puissants personnages et s'assignant des objectifs précis et limités (orphelines, repenties...).

C'est sur ces bases que le milieu du siècle voit " l'épanouissement de la spiritualité active " ( $3^{\mathrm{e}}$ partie) avec la véritable " révolution de la charité " (pour reprendre l'expression de l'auteur) qu'engage Vincent de Paul : l'assistance aux pauvres n'est plus d'abord, et seulement, un chemin de progression spirituelle personnelle - l'occasion pour le riche de faire son salut, vision traditionnelle qu'exprime encore à cette époque un Saint-Cyran - mais une réponse à une situation concrète où derrière le pauvre se profile le Dieu de l'incarnation. Une telle modification de perspective suppose des moyens accrus et une organisation nouvelle : celle-ci repose moins sur des aventures individuelles que sur l'action collective de petits groupes, à l'exemple de celui qui fonde les Incurables de Paris en 1632. Ces groupes sont presque tous féminins : à l'image des Dames de la Charité (1634), on y trouve des filles dévotes groupées autour d'une veuve, sans vœux ni statut juridique officiel, sous le patronage du curé de la paroisse ou du réseau de Monsieur Vincent. Vers 1640, les principales paroisses de Paris se sont dotées, sur ce modèle, de compagnies de charité visitant à domicile un nombre de malades comparable à ceux qui peuplent l'Hôtel-Dieu (lui-même pris en charge par des religieuses réformées).

C'est dans ce contexte que survient le traumatisme de la Fronde ( $4^{\mathrm{e}}$ partie). Face au cortège démultiplié des misères, l'aumône s'impose avec une urgence inédite. Les casuistes en débattent : pour certains, le refus de l'aumône est péché mortel en cas de nécessité extrême, quand la vie du prochain est en jeu; d'autres sont plus laxistes, concédant que nul n'est tenu, parmi les laïcs en tout cas, de donner sur ce qui lui est nécessaire (un nécessaire parfois largement entendu). Cette position accommodante tend sans nul doute à l'emporter avec le temps, mais seulement après que soit retombée l'exceptionnelle conjoncture charitable des années 1651-1655. S'est en effet déployée alors la " première grande campagne caritative d'opinion ", reposant sur un usage du petit imprimé qui n'est pas sans rappeler les contemporaines Mazarinades : une trentaine de "relations " 
imprimées ont été généreusement diffusées, appelant à la générosité en faveur des régions les plus touchées des environs de Paris. Elles mobilisent différents réseaux : proches de Vincent de Paul, jansénistes, Compagnie du SaintSacrement... réunis pour la dernière fois. Cet effort " médiatique " n'est pas sans quelques résultats, comme en témoigne la comptabilité privée de Maignard de Bernières, mais il s'interrompt à la fin 1655, moins parce que les misères de la Fronde ont disparu que parce qu'une nouvelle phase de l'assistance s'est ouverte, reposant sur des structures qui, d'une manière ou d'une autre, tendent à s'institutionnaliser : les Dames de la Charité, réunion informelle de bonnes volontés individuelles, cèdent le pas aux Filles de la Charité érigées en " institut séculier ", prononçant donc des vœux, même si ceux-ci demeurent simples et renouvelables; dans les paroisses, une nouvelle génération de compagnies de charité poursuit désormais des objectifs qui sont d'ordre moral au moins autant que de stricte assistance; et surtout, le vieux projet d'un " hôpital général ", évoqué dans les années 1610, resurgit face à la prolifération de la mendicité. Son but est au fond de "renfermer " tous ceux qui échappaient jusqu'alors à la multitude des institutions particulières préexistantes. Le 7 mai 1657, l'Hôpital général de Paris ouvre ses portes - ou les ferme, comme on voudra - sur quelques 5000 pauvres dispersés sur cinq sites, comprenant surtout des enfants et des vieillards.

C'est dire que l'assistance est désormais entrée dans le " temps des institutions" ( $5^{\text {e }}$ partie). Voici désormais le temps de la charité calculée : l'auteur le démontre à partir de l'analyse des legs testamentaires aux hôpitaux, notamment à l'Hôtel Dieu qui ne reçoit pas moins de 149 legs universels, presque toujours entre 1640 et 1720 . Le profil des donateurs est large : de grandes familles aristocratiques, des manieurs d'argent gravitant dans les cercles du pouvoir, quelques maîtres-artisans aussi, pour la plupart sans héritiers directs. Pour tous, " le dosage est subtil entre l'intérêt personnel, la charité et les droits des héritiers ". Certains ne s'interdisent pas de transporter en faveur des hôpitaux de vieilles rentes mal assurées; d'autres imposent, en contrepartie de leur donation, le versement d'une pension viagère qui garantisse leurs vieux jours. Ce qui rend périlleuse, on s'en doute, la bonne gestion de ces hôpitaux devant désormais acquitter une multitude de petites rentes : l'État monarchique s'en émeut et impose en 1690 de drastiques mesures de redressement aux Incurables et à l'Hôtel-Dieu.

Car l'alourdissement du rôle de l'État est indissociable de cette "rationalisation " de la charité : le temps des institutions est aussi le "temps du roi ", qui veille à la rectitude des finances hospitalières et oriente aussi plus fermement la politique d'assistance dans le sens de la répression de la mendicité. En 1701, l'Hôpital général de Paris accueille toujours beaucoup d'enfants, et même de plus en plus d'enfants abandonnés (selon l'auteur, la pratique commencerait de se banaliser entre 1660 et 1700) mais on y trouve des bataillons croissants de mendiant(e)s et de filles internées, mises au travail forcé, sans perspective de correction ou de réintégration. La charité proprement dite se ressent de ce poids croissant de l'État qui a pour effet de casser l'élan des initiatives individuelles : la crise de 1693-1694 ne suscite rien qui ressemble à l'élan de la Fronde : " Les ateliers publics sont des initiatives royales, le pain ou l'argent distribué est l'argent ou le pain du roi " (p. 288). Cette relativisation de la charité privée n'est pas sans échos dans la littérature spirituelle du temps, dont le best-seller porte le titre révélateur du "Chrétien intérieur ". Rien de plus instructif, remarque J. Depauw, que de comparer, à quelques années de distance, deux caricatures de dévots, Tartuffe de Molière et Onuphre de La Bruyère : Tartuffe va aux prisonniers ou aux hôpitaux, multipliant les aumônes personnelles; Onuphre pré- 
fère l'église paroissiale à l'Hôtel-Dieu, fait retraite plus que charité, lit surtout des œuvres spirituelles où la dévotion se veut intérieure et discrète. Et La Bruyère raille volontiers la mondanité du "sermon de charité ", comme si la rhétorique sacrée était devenue l'ultime refuge du "formidable élan de solidarité " - comme on dit aujourd'hui - des décennies précédentes.

On mesure à ce bref résumé la richesse d'un ouvrage à la progression convaincante, où de fermes conclusions viennent résumer à propos une démonstration parfois tortueuse dans le détail de son argumentation. Certaines imperfections formelles, notamment dans le renvoi aux annexes, n'affectent pas l'intérêt de ce qui est sans nul doute une étude de référence sur la "sensibilité charitable ". De grande importance pour l'histoire de Paris, ce livre dépasse évidemment aussi l'horizon de la capitale : d'abord parce que celle-ci est un révélateur privilégié d'évolutions plus générales, mais aussi parce que la littérature de spiritualité analysée par l'auteur n'est parisienne que par son lieu d'édition; elle a en réalité connu une audience bien plus large. On se gardera pourtant d'ériger le cas parisien en modèle unique, ce que l'auteur n'entend nullement faire : la Bretagne, bien connue par les travaux d'A. Croix, J.-L. Bruzulier ou G. Haudebourg, est là pour rappeler la force de l'attachement à «l'aumône manuelle " et sa durable résistance à la " charité institutionnelle ".

Georges Provost

Blanc-Chaleard, Marie-Claude, Douli, Caroline, Dyonet, Nicole et Milliot, Vincent (textes réunis et présentés par), Police et migrants, France 1667-1939, actes du colloque " Police et migrants, France 1667-1945 " organisé à l'université d'Orléans les 28 et 29 octobre 1999, Rennes, PUR, 2001.

Le colloque organisé à l'université d'Orléans en octobre 1999 posait une question relativement nouvelle mais perspicace : la police, au sens contemporain du terme, pousse-t-elle une partie de ses racines historiques dans le contrôle, non des populations résidentes, mais dans celui des migrants, des étrangers, des nomades, supposés représenter l'inconnu, I'inhabituel et donc le danger?

Les actes ici rassemblés pourraient incliner à répondre oui : "La mobilité apparaît comme l'un des moteurs de la réflexion sur la police générale et particulière et sur l'inflexion de ses démarches qui entrainent les comportements de tous " (Daniel Roche, Introduction, p. 31). Certes, on a par ailleurs la preuve que la police possède aussi d'autres origines : espionnage et renseignement politique, protection des puissants, lutte contre les opposants, maintien des fonctions de base de la cité. Reste que la démonstration est ici bien faite qu'une des voies de développement de la police a reposé sur le contrôle, la mise à l'écart ou l'expulsion des corps étrangers. La frontière entre ces différentes catégories d'activités reste par ailleurs floue et perméable.

Un certain nombre de constantes apparaissent dans des articles pourtant divers, même si l'évolution des mœurs et des techniques peut infléchir la forme de ces invariants :

- Les buts avoués du contrôle : le migrant est toujours vu comme un élément hors des normes dans un État policé (aux deux sens du terme justement). Le bon sujet (ou citoyen) ne se déplace pas. Partir est toujours la manifestation d'une insatisfaction ou de pulsions dangereuses. "On retrouve toujours, dans les représentations, les mêmes soupçons attachés aux errants et nomades : la concur- 